\title{
Clinically isolated neurosarcoidosis: A recommended diagnostic path
}

\author{
Wegener, Susanne ; Linnebank, Michael ; Martin, Roland ; Valavanis, Anton ; Weller, Michael
}

\begin{abstract}
The involvement of the central nervous system in sarcoidosis can manifest with a variety of neurological symptoms, most of them nonspecific. We identified 13 patients with neurosarcoidosis diagnosed at our clinic. Six of 13 patients presented with clinically isolated neurosarcoidosis (CINS) without signs or symptoms of systemic disease. CINS patients were not different with respect to age, as well as imaging and spinal fluid findings, or disease course. However, we found spinal cord involvement in neurosarcoidosis patients much more common than previously described (in 8 out of 13 patients). Spinal cord affection was associated with older age at diagnosis and a less favorable response to therapy. Based on our findings, we propose a diagnostic path for neurosarcoidosis, including spinal magnetic resonance imaging (MRI) as a mandatory and early step during diagnostic workup.
\end{abstract}

DOI: https://doi.org/10.1159/000366199

Posted at the Zurich Open Repository and Archive, University of Zurich

ZORA URL: https://doi.org/10.5167/uzh-101284

Journal Article

Published Version

Originally published at:

Wegener, Susanne; Linnebank, Michael; Martin, Roland; Valavanis, Anton; Weller, Michael (2015). Clinically isolated neurosarcoidosis: A recommended diagnostic path. European Neurology, 73(1-2):7177.

DOI: https://doi.org/10.1159/000366199 


\title{
Clinically Isolated Neurosarcoidosis: A Recommended Diagnostic Path
}

\author{
Susanne Wegener ${ }^{\mathrm{a}}$ Michael Linnebank ${ }^{\mathrm{a}}$ Roland Martin ${ }^{\mathrm{a}} \quad$ Anton Valavanis $^{\mathrm{b}}$ \\ Michael Weller ${ }^{\mathrm{a}}$ \\ ${ }^{a}$ Department of Neurology, and ${ }^{b}$ Department of Neuroradiology, University Hospital Zurich, Switzerland
}

\section{Key Words}

Neurosarcoidosis - Sarcoidosis - Magnetic resonance imaging $\cdot$ Spinal fluid analysis

\begin{abstract}
The involvement of the central nervous system in sarcoidosis can manifest with a variety of neurological symptoms, most of them nonspecific. We identified 13 patients with neurosarcoidosis diagnosed at our clinic. Six of 13 patients presented with clinically isolated neurosarcoidosis (CINS) without signs or symptoms of systemic disease. CINS patients were not different with respect to age, as well as imaging and spinal fluid findings, or disease course. However, we found spinal cord involvement in neurosarcoidosis patients much more common than previously described (in 8 out of 13 patients). Spinal cord affection was associated with older age at diagnosis and a less favorable response to therapy. Based on our findings, we propose a diagnostic path for neurosarcoidosis, including spinal magnetic resonance imaging (MRI) as a mandatory and early step during diagnostic workup.
\end{abstract}

(c) 2014 S. Karger AG, Basel (c) 2014 S. Karger AG, Base

0014-3022/14/0732-0071\$39.50/0

\section{Introduction}

Sarcoidosis, a multisystem disease characterized by noncaseating granulomas predominantly affecting the lungs, is not uncommon in Northern European countries, with an annual incidence of 5-40 per 100,000 [1]. The etiology of sarcoidosis remains unknown, but an autoimmune reaction that is triggered by a pathogen (not yet known) in genetically predisposed individuals is the most likely cause. Neurosarcoidosis refers to disease manifestation of sarcoidosis within the nervous system. It is clinically apparent in $5-10 \%$ of patients, but has been demonstrated in up to $25 \%$ of sarcoidosis patients at autopsy [2-4]. The histopathological presentation of neurosarcoidosis is either the pathognomonic noncaseating granuloma with lymphomonuclear infiltrates and multinuclear macrophages, or an infiltration of inflammatory cells into the meninges or extracellular spaces causing microvascular damage and diffuse white matter lesions [57]. Neurological symptoms may range from basal meningitis with cranial nerve involvement to limb paralysis or seizures due to a granulomatous lesion or even slowly

\section{KARGER 125}

E-Mail karger@karger.com www.karger.com/ene
Susanne Wegener, MD

Department of Neurology

University Hospital Zurich, Frauenklinikstrasse 26

CH-8091 Zurich (Switzerland)

E-Mail Susanne.Wegener@usz.ch 
Table 1. Clinical characteristics of patients with neurosarcoidosis

\begin{tabular}{|c|c|c|c|c|c|c|c|c|}
\hline $\begin{array}{l}\text { Patient } \\
\text { no./sex }\end{array}$ & $\begin{array}{l}\text { Age, } \\
\text { years }\end{array}$ & $\begin{array}{l}\text { Neurological symptoms } \\
\text { (presenting, y/n) }\end{array}$ & $\begin{array}{l}\text { Systemic } \\
\text { symptoms }\end{array}$ & cMRI & sMRI & LP & Treatment & $\begin{array}{l}\text { Clinical course } \\
\text { (follow up, m) }\end{array}$ \\
\hline $1 / M^{* *}$ & 35 & Cranial neuritis (y) & + & + & n.d. & + & Steroids + MTX & better (40) \\
\hline $2 / \mathrm{M}^{* *}$ & 49 & Headache, focal seizure (n) & + & + & + & - & Steroids & stable (30) \\
\hline $3 / \mathrm{M}^{* *}$ & 57 & $\begin{array}{l}\text { Meningitis, optic neuritis, } \\
\text { headache, gait disturbance (n) }\end{array}$ & + & + & + & + & $\begin{array}{l}\text { Steroids, steroids + MTX, } \\
\text { steroids }\end{array}$ & worse (39) \\
\hline $5 / \mathrm{F}^{* *}$ & 46 & Paraplegia (Myelitis) (n) & + & - & + & + & Steroids + MTX & died (37) \\
\hline $6 / \mathrm{F}^{* *}$ & 27 & Polyneuritis (y) & + & - & n.d. & + & None & better (33) \\
\hline $7 / \mathrm{F}^{* * *}$ & 31 & Optic neuritis $(y)$ & + & + & + & + & $\begin{array}{l}\text { Steroids for } 144 \mathrm{~m}, \text { MTX } \\
\text { monotherapy since } 24 \mathrm{~m}\end{array}$ & worse (169) \\
\hline $10 / M^{*}$ & 20 & Basal meningitis (y) & - & + & n.d. & + & Steroids + mycophenolate & stable (204) \\
\hline $11 / \mathrm{F}^{* *}$ & 38 & Progressive left paresis/radiculitis (y) & - & - & + & + & $\begin{array}{l}\text { Steroids } 17 \mathrm{~m} \text {, MTX } 3 \mathrm{~m} \\
\text { stopped due to stabilization of } \\
\text { disease, infliximab } 3 \mathrm{~m} \text {, MTX } \\
13 \mathrm{~m} \text {, Rituximab } 6 \mathrm{~m} \text {, tocili- } \\
\text { zumab } 3 \mathrm{~m} \text {, steroids }\end{array}$ & worse (62) \\
\hline $12 / \mathrm{M}^{* *}$ & 52 & Basal meningitis (y) & - & + & n.d. & + & Steroids & stable (2) \\
\hline $13 / \mathrm{M}^{* *}$ & 51 & Tetraparesis (y) & - & - & + & + & Steroids & stable (4) \\
\hline
\end{tabular}

Diagnostic certainty: Neurosarcoidosis possible*, probable**, certain*** $+=$ Pathological findings; $-=$ normal; n.d. $=$ not done. cMRI = cranial MRI; sMRI = MRI of the spine; $\mathrm{LP}=$ lumbar puncture results; $\mathrm{MTX}=$ methotrexate; $\mathrm{m}=$ months.

progressive cognitive decline [8]. Due to the variability of symptoms, neurosarcoidosis is a diagnostic challenge. Furthermore, there are currently no unambiguous (imaging or laboratory) parameters to aid in the diagnosis.

We here report on 13 neurosarcoidosis cases from our institution with the typical manifold neurological symptomatology. Based on our experience, we recommend a diagnostic path to aid in identifying the disease.

\section{Patients and Methods}

We searched the clinical database of the Department of Neurology at the University Hospital Zurich for all patients that were seen with suspected neurosarcoidosis since 2000. All cases with a diagnostic certainty of at least 'possible neurosarcoidosis' [9] were included. 'Possible neurosarcoidosis' was defined as clinical condition and neurodiagnostic workup consistent with neurosarcoidosis, but without histological evidence. For 'probable neurosarcoidosis', typical laboratory findings including cerebrospinal fluid (CSF) abnormalities (elevated levels of CSF protein and/or cells, presence of oligoclonal bands) and/or histological evidence of systemic disease had to be present in addition to the clinical and neurodiagnostic findings compatible with neurosarcoidosis, while the diagnosis of 'definite neurosarcoidosis' required proof by a nervous system biopsy. Patient charts were reviewed and clinical characteristics, imaging findings, results of laboratory tests, as well as treatment and outcome were recorded. Comparisons between groups were done using a two-sided $t$ test. Furthermore, a Medline search for 'spinal cord sarcoidosis' was performed and all studies from 1994 onwards reporting more than one case of spinal cord sarcoidosis were identified. All papers reporting clinical outcome or spinal cord MRI data were included for the analysis in table 3.

\section{Results}

\section{Patient Characteristics}

Thirteen patients were diagnosed with neurosarcoidosis between 2000 and 2014, of those 1 with possible, 11 with probable, and 1 with definite neurosarcoidosis. Patient characteristics are shown in table 1 . The mean age at 
Table 2. Imaging and spinal fluid/serum findings in patients with neurosarcoidosis

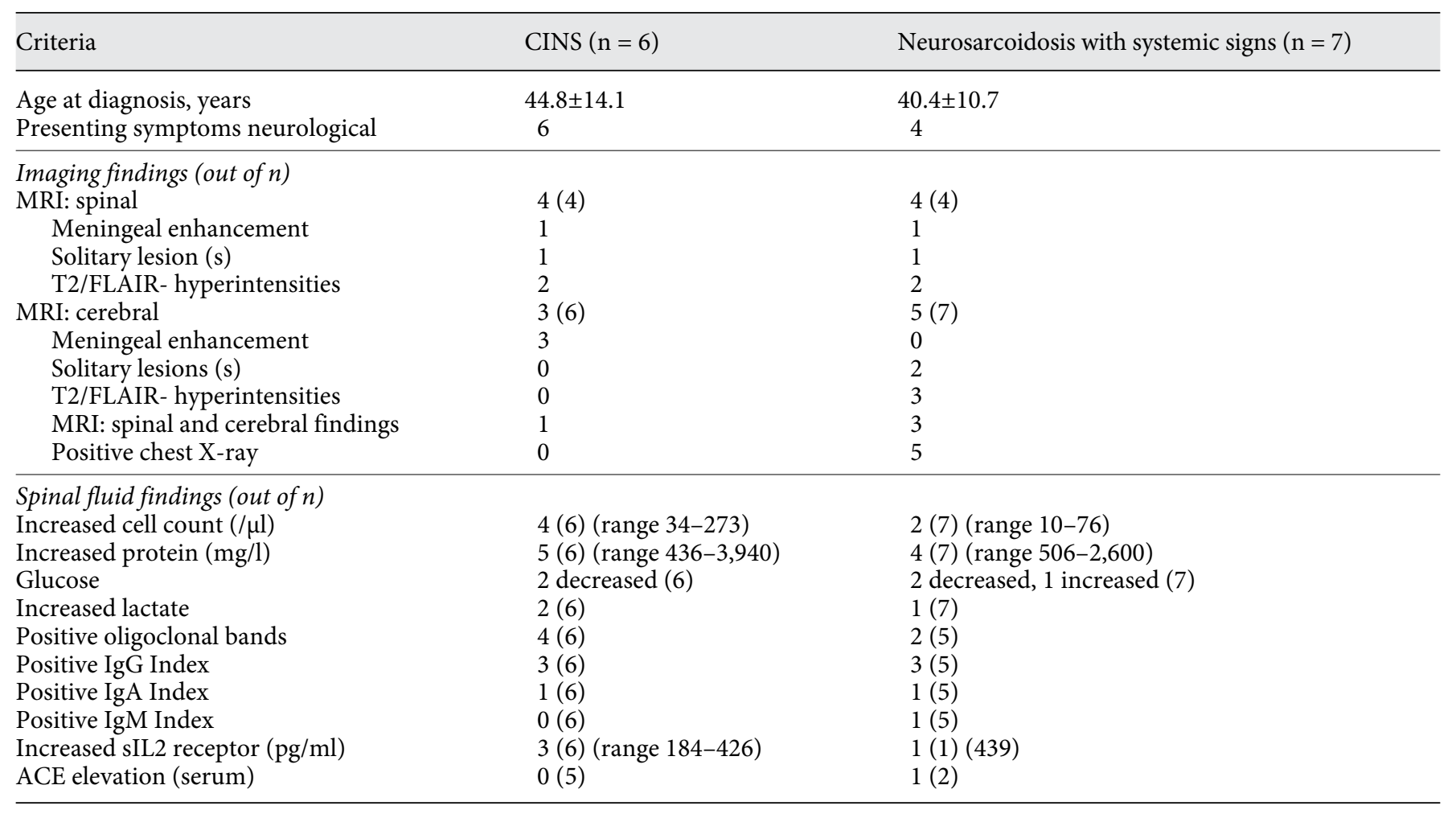

the onset of neurological symptoms was 42.5 years (range $20-60$ years), with a male:female ratio of close to $1: 1$. The median follow-up was 37 months. Seven patients displayed clinical signs of systemic sarcoidosis before or at the time of presentation for neurological workup, 6 with pulmonary disease and 1 with cardiac arrhythmia that led to further diagnostic workup and was attributed to sarcoidosis. Four of these 7 patients presented initially with neurological symptoms, but were diagnosed only after the occurrence of systemic signs. The remaining $6 \mathrm{pa}-$ tients were diagnosed only based on neurological symptoms (P8-13 in table 1), and will be referred to as clinically isolated neurosarcoidosis (CINS). CSF was abnormal in 12 of 13 cases with findings ranging from mild pleocytosis to substantial elevation in protein content, positive oligoclonal bands, and/or increased levels of soluble interleukin 2 receptor alpha chain (CD25) (tables 1 and 2).

Neuropathy was suspected in one patient who reported a tingling sensation in both lower legs. ENMG was unremarkable in this case. In two patients, one of them only several years after diagnosis of neurosarcoidosis and immunosuppressive treatment, ENMG revealed a beginning axonal peripheral neuropathy.

Clinically Isolated Neurosarcoidosis
Except for one case (P6) with rapid spontaneous clinical improvement, all patients received immunosuppressive/anti-inflammatory therapies, either with steroids alone (P2, P4, P8, P12, P13) at an earlier stage of treatment with rather short follow-up: median 30 months; steroids in combination with methotrexate (MTX): P1, P3, P5 with a median follow-up of 39 months, or in combination with other drugs: azathioprine (P9), mycophenolate (P10), or monotherapy with MTX (P7). One patient with severe disease progression was switched from steroids to MTX and later to infliximab and tocilizumab, without achieving sufficient control of symptoms (P11). Overall, the clinical course was good (neurological signs/ symptoms stable or better) in 9 patients and progressive (worsening of signs/symptoms) in 4; one patient died following long-term steroid therapy and immobilization.

\section{Imaging Findings}

MRI of the brain was abnormal in 8 cases and normal in 5 . MRI of the spinal cord was pathological in 8 cases, not done in 4 cases and normal in only 1 patient (table 1 ). Both, spinal and cerebral imaging findings were present in 5 patients. Patients with spinal cord involvement tend- 

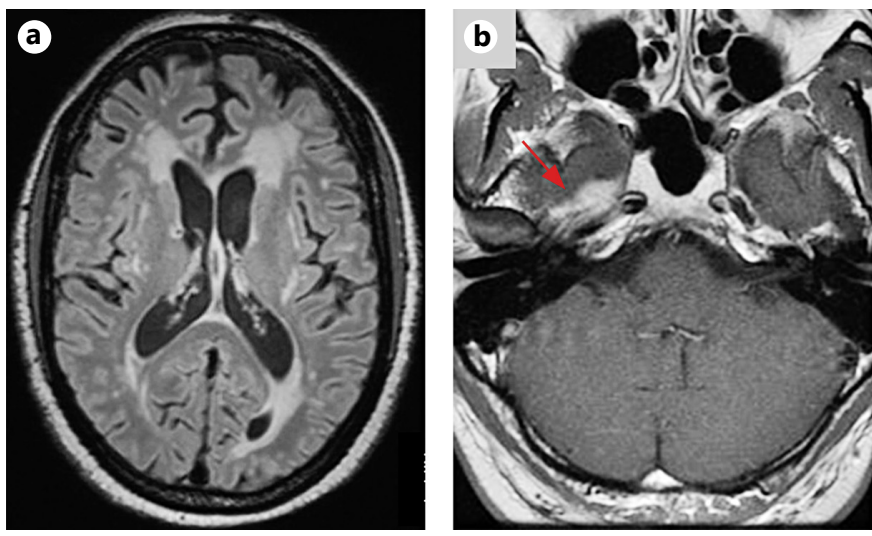

Fig. 1. Neurosarcoidosis imaging patterns. Characteristic MRI findings in neurosarcoidosis patients: (a) T2/ FLAIR hyperintensities without contrast enhancement, (b) brain solitary, contrast enhancing

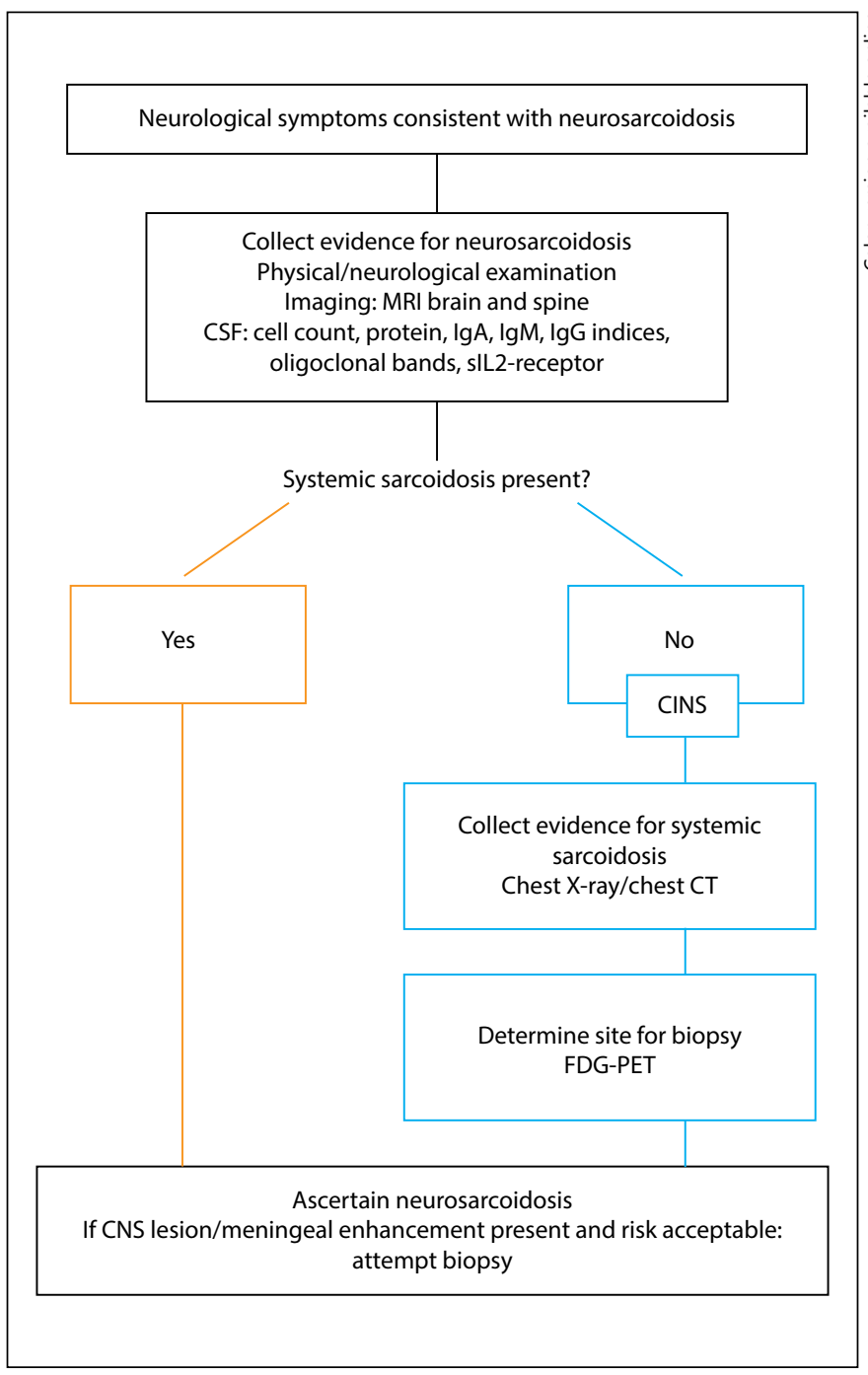

Fig. 2. Suggested diagnostic path for neurosarcoidosis.
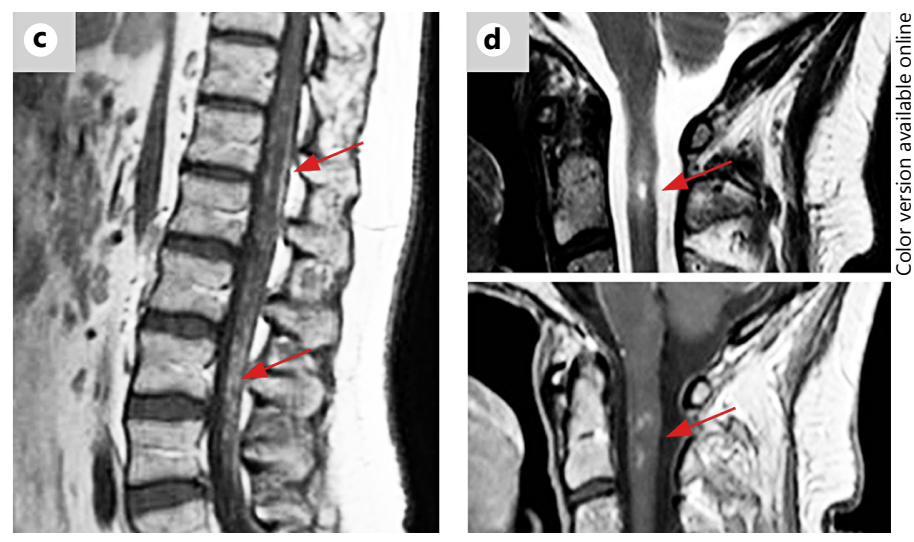

lesion (arrow), (c) meningeal and diffuse parenchymal contrast enhancement (arrows), and (d) spinal cord solitary contrast enhancing lesion (upper panel: T2w image, lower panel: T1 post contrast image).

ed to be older ( $48.8 \pm 9.2$ vs. $36.2 \pm 5.1$ years, $p=0.06)$ and had a less favorable response to therapy $(\mathrm{p}=0.01)$.

We further classified MRI patterns of neurosarcoidosis into a) meningeal contrast enhancement, b) solitary lesions, or c) predominantly subcortical T2/FLAIR hyperintensities with nonspecific appearance (table 2 and fig. 1). These three patterns could be discerned on MRI of the brain as well as the spine. All of the solitary spinal lesions/T2 hyperintensities $(n=6)$ were located in the cervical or thoracic spine.

Chest X-ray did not show any abnormalities in patients with CINS, but was positive in 5 out of the remaining 7 patients with systemic symptoms (table 2). In 4 out of the 6 CINS patients, FDG-PET was performed to search for a biopsy site, which was successful in all 4 . In 3 of these patients, the histological confirmation of sarcoidosis was based on biopsy results from FDG-enhancing sites revealed by PET.

\section{Discussion}

To reach the diagnosis of definite or at least probable neurosarcoidosis, a biopsy must be obtained to demonstrate the typical histological picture of sarcoidosis either in the nervous system (definite) or another affected organ along with imaging and laboratory signs suggestive of neurosarcoidosis (probable neurosarcoidosis). In patients with known systemic sarcoidosis and neurological symptoms that remain otherwise unexplained, the suspicion of neurosarcoidosis is high. The diagnostic path for this scenario is focused on the collection of evidence for CNS involvement by clinical examination, MRI, and CSF studies (fig. 2). It is now established that neither serum 
Table 3. Summary of studies reporting spinal cord affection in neurosarcoidosis

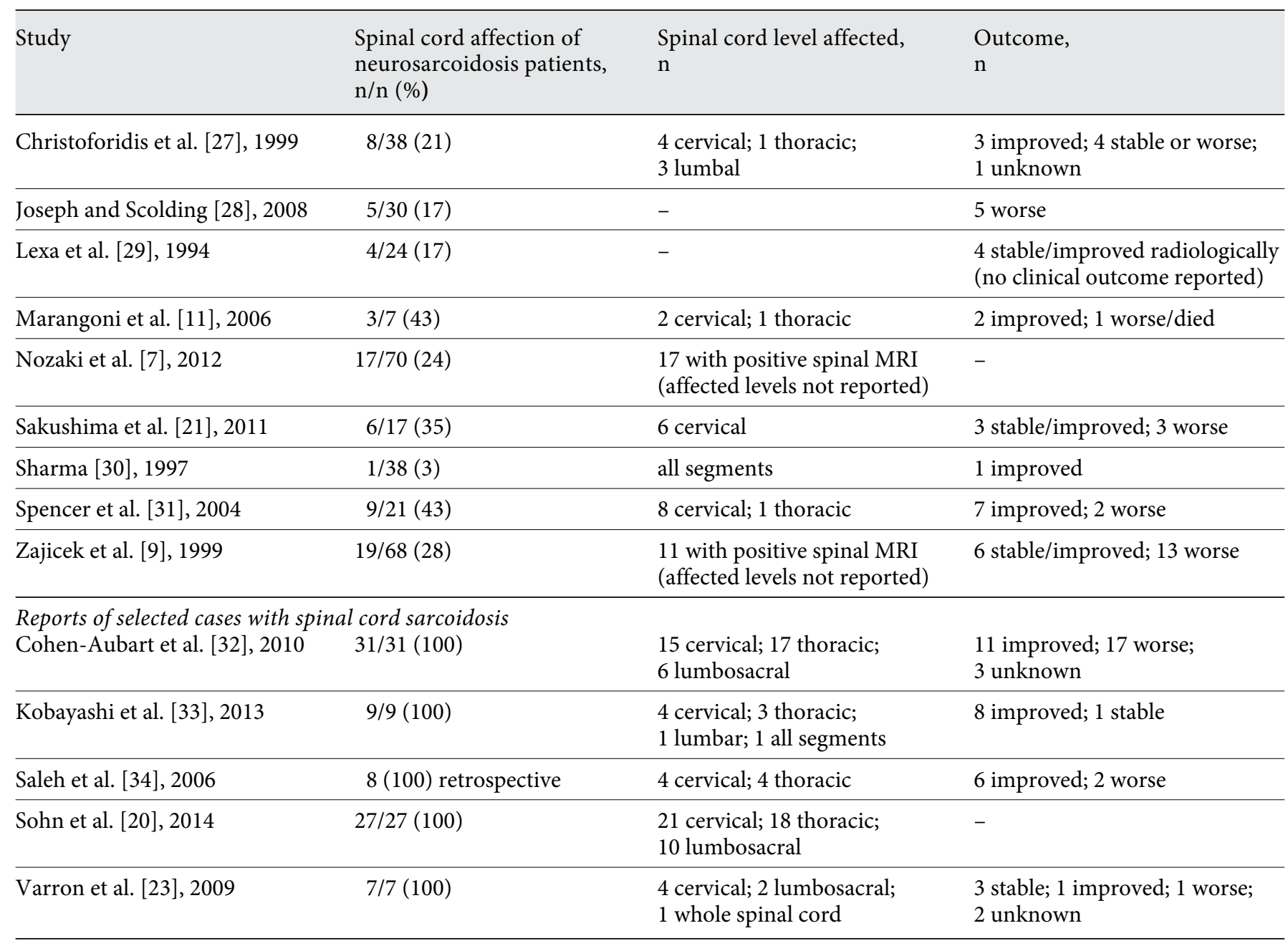

Not reported: ‘-’.

nor spinal fluid angiotensin converting enzyme (ACE) levels are helpful for the diagnosis of neurosarcoidosis (sensitivity of 24-55\% for CSF levels) [10, 11]. CSF analysis should include glucose, lactate, protein, number and types of cells and antibody indices (IgM, IgA, IgG) since these parameters may correlate with disease activity [12]. However, depending on the MRI-defined disease extension (e.g., diffuse leptomeningeal enhancement versus solitary lesions) and disease activity, CSF parameters might be normal in approximately $40 \%$ of cases [12]. Oligoclonal bands were positive only in $22 \%$ of cases with probable or definite neurosarcoidosis [12]. Spinal fluid quantification of soluble CSF interleukin 2 receptor alpha chain, a marker of T-cell activation, appears suitable in the diagnostic workup for neurosarcoidosis, since levels above $150 \mathrm{pg} / \mathrm{ml}$ allow to discriminate neurosarcoidosis from other differential diagnoses such as multiple sclerosis and vasculitis as well as healthy controls with an overall accuracy of $93 \%$ (sensitivity of $61 \%$ and specificity of 93\%) [13]. While activated CD4+ T- cells are the hallmark of the sarcoid granuloma immunopathogenesis and the CD4/CD8 T-cell ratio typically increased in bronchoalveolar lavage of sarcoidosis patients, the diagnostic utility of this test for neurosarcoidosis remains uncertain. In a previous case series of 8 patients, it was increased only in three and normal in five [14-16]. Sarcoid-induced neuropathy is rare, but may be a presenting symptom of neurosarcoidosis [3, 17-19]. ENMG, albeit not specific for neurosarcoidosis, is certainly of value in cases in which symptoms of neuropathy or myopathy are reported and 
for diagnosis and follow up of therapy-induced myopathy or neuropathy. Besides the above-mentioned diagnostic parameters, neuroimaging with MRI remains an essential part of the diagnostic workup for neurosarcoidosis. The MRI protocol should include high resolution T2 and FLAIR images for detection of edema or white matter changes, as well as pre- and post-contrast T1, to reveal even subtle meningeal contrast enhancement. To discriminate vascular pathologies or microbleeds, MRA and susceptibility-weighted (T2*) imaging should also be performed.

The prevalence of spinal cord lesions on MRI in our patient cohort was high (62\%). Previous reports estimated the prevalence of spinal cord neurosarcoidosis to be about $1 \%$ of all sarcoidosis patients, or between 3 and $43 \%$ of neurosarcoidosis patients (table 3) [20-22]. We assume that spinal cord involvement is often missed, either due to unspecific symptoms that might be related to cord involvement such as sensory disturbances or because it was clinically silent. The cervical spine is most frequently affected; however, all spinal cord levels may reveal disease activity (table 3 ). Identification of spinal cord involvement is important, since it might indicate patients with a more protracted disease course requiring earlier start of nonsteroidal therapies [21, 23, 24]. While our case series supports a more unfavorable clinical course when the spinal cord is affected, it is interesting to note that clinical outcome data of previous studies in this subgroup of patients are actually less clear (table 3 ). Due to the signifi- cant prevalence of spinal cord involvement, we suggest that spinal MRI should be included in the routine workup of neurosarcoidosis. Since the course of treatment may last for prolonged periods of time, imaging evidence of disease activity is highly important and can also monitor treatment efficacy. A CNS biopsy should be undertaken to clear all doubts, if any.

Due to the rarity of the disease and the small sample size of this retrospective study, interpretation of our findings is limited. However, based on our experience, we suggest the following diagnostic path for patients with suspected neurosarcoidosis.

In the case of a patient presenting with neurological symptoms consistent with neurosarcoidosis, but no further clinical evidence of systemic sarcoidosis (fig. 2, right side), searching for systemic disease manifestations is the first step. One aspect is that subclinical systemic disease should be recognized; another is that a biopsy site might be found. Routine workup for systemic involvement should include a chest-CT scan, and, if doubts remain, [18F]-fluorodeoxyglucose-positron emission tomography, as it might be more sensitive to reveal a suspicious lymph node that is accessible at minimal procedural risk $[25,26]$.

\section{Disclosure Statement}

The authors have no conflict of interest to disclose.

\section{References}

1 Pietinalho A, Hiraga Y, Hosoda Y, Lofroos $\mathrm{AB}$, Yamaguchi M, Selroos O: The frequency of sarcoidosis in Finland and Hokkaido, Japan. A comparative epidemiological study. Sarcoidosis 1995; 12:61-67.

2 Stern BJ, Krumholz A, Johns C, Scott P, Nissim J: Sarcoidosis and its neurological manifestations. Arch Neurol 1985;42:909-917.

3 Gascon-Bayarri J, Mana J, Martinez-Yelamos S, Murillo O, Rene R, Rubio F: Neurosarcoidosis: report of 30 cases and a literature survey. Eur J Intern Med 2011;22:e125-e132.

4 Iannuzzi MC, Rybicki BA, Teirstein AS: Sarcoidosis. N Engl J Med 2007;357:2153-2165.

5 Segal BM: Neurosarcoidosis: diagnostic approaches and therapeutic strategies. Curr Opin Neurol 2013;26:307-313.

6 Gonzalez-Aramburu I, Ruiz-Perez E, GomezRoman J, Quirce R, Larrosa D, Pascual J: Sarcoidosis presenting as transient ischemic attack status. J Stroke Cerebrovasc Dis 2012;21: 515-517.
7 Nozaki K, Scott TF, Sohn M, Judson MA: Isolated neurosarcoidosis: case series in 2 sarcoidosis centers. Neurologist 2012;18:373377.

8 Spiegel DR, Morris K, Rayamajhi U: Neurosarcoidosis and the complexity in its differential diagnoses: a review. Innov Clin Neurosci 2012;9:10-16.

9 Zajicek JP, Scolding NJ, Foster O, et al: Central nervous system sarcoidosis - diagnosis and management. QJM 1999;92:103-117.

10 Khoury J, Wellik KE, Demaerschalk BM, Wingerchuk DM: Cerebrospinal fluid angiotensin-converting enzyme for diagnosis of central nervous system sarcoidosis. Neurologist 2009;15:108-111.

11 Marangoni S, Argentiero V, Tavolato B: Neurosarcoidosis. Clinical description of 7 cases with a proposal for a new diagnostic strategy. J Neurol 2006;253:488-495.

12 Wengert O, Rothenfusser-Korber E, Vollrath B, et al: Neurosarcoidosis: correlation of cere- brospinal fluid findings with diffuse leptomeningeal gadolinium enhancement on MRI and clinical disease activity. J Neurol Sci 2013; 335:124-130.

13 Petereit HF, Reske D, Tumani H, et al: Soluble CSF interleukin 2 receptor as indicator of neurosarcoidosis. J Neurol 2010;257:1855-1863.

14 Danila E, Norkuniene J, Jurgauskiene L, Malickaite R: Diagnostic role of BAL fluid CD4/ CD8 ratio in different radiographic and clinical forms of pulmonary sarcoidosis. Clin Respir J 2009;3:214-221.

15 Stern BJ, Griffin DE, Luke RA, Krumholz A, Johns CJ: Neurosarcoidosis: cerebrospinal fluid lymphocyte subpopulations. Neurology 1987;37:878-881.

16 Reske D, Petereit HF, Heiss WD: Difficulties in the differentiation of chronic inflammatory diseases of the central nervous system - value of cerebrospinal fluid analysis and immunological abnormalities in the diagnosis. Acta Neurol Scand 2005;112:207-213. 
17 Burns TM, Dyck PJ, Aksamit AJ, Dyck PJ: The natural history and long-term outcome of 57 limb sarcoidosis neuropathy cases. J Neurol Sci 2006;244:77-87.

18 Gainsborough N, Hall SM, Hughes RA, Leibowitz S: Sarcoid neuropathy. J Neurol 1991; 238:177-180.

19 Hoitsma E, Faber CG, Drent M, Sharma OP: Neurosarcoidosis: a clinical dilemma. Lancet Neurol 2004;3:397-407.

20 Sohn M, Culver DA, Judson MA, Scott TF, Tavee J, Nozaki K: Spinal cord neurosarcoidosis. Am J Med Sci 2014;347:195-198.

21 Sakushima K, Yabe I, Nakano F, et al: Clinical features of spinal cord sarcoidosis: analysis of 17 neurosarcoidosis patients. J Neurol 2011; 258:2163-2167.

22 Duhon BS, Shah L, Schmidt MH: Isolated intramedullary neurosarcoidosis of the thoracic spine: case report and review of the literature. Eur Spine J 2012;21(suppl 4):S390-S395.

23 Varron L, Broussolle C, Candessanche JP, et al: Spinal cord sarcoidosis: report of seven cases. Eur J Neurol 2009;16:289-296.
24 Bradley DA, Lower EE, Baughman RP: Diagnosis and management of spinal cord sarcoidosis. Sarcoidosis Vasc Diffuse Lung Dis 2006; 23:58-65.

25 Meenakshi M, Arnold C, Broadley SA: The value of [18f]-fluorodeoxyglucose-positron emission tomography/CT scanning in the diagnosis of neurosarcoidosis. J Clin Neurosci 2012;19:1461-1462.

26 Bolat S, Berding G, Dengler R, Stangel M, Trebst C: Fluorodeoxyglucose positron emission tomography (FDG-PET) is useful in the diagnosis of neurosarcoidosis. J Neurol Sci 2009;287:257-259.

27 Christoforidis GA, Spickler EM, Recio MV, Mehta BM: MR of CNS sarcoidosis: correlation of imaging features to clinical symptoms and response to treatment. AJNR Am J Neuroradiol 1999;20:655-669.

28 Joseph FG, Scolding NJ: Neurosarcoidosis: a study of 30 new cases. J Neurol Neurosurg Psychiatry 2009;80:297-304.

29 Lexa FJ, Grossman RI: MR of sarcoidosis in the head and spine: spectrum of manifestations and radiographic response to steroid therapy. AJNR Am J Neuroradiol 1994;15: 973-982.
30 Sharma OP: Neurosarcoidosis: a personal perspective based on the study of 37 patients. Chest 1997;112:220-228.

31 Spencer TS, Campellone JV, Maldonado I, Huang N, Usmani Q, Reginato AJ: Clinical and magnetic resonance imaging manifestations of neurosarcoidosis. Semin Arthritis Rheum 2004;34:649-661.

32 Cohen-Aubart F, Galanaud D, Grabli D, et al: Spinal cord sarcoidosis: clinical and laboratory profile and outcome of 31 patients in a case-control study. Medicine (Baltimore) 2010;89:133-140.

33 Kobayashi S, Nakata W, Sugimoto H: Spinal magnetic resonance imaging manifestations at neurological onset in Japanese patients with spinal cord sarcoidosis. Intern Med 2013;52:2041-2050.

34 Saleh S, Saw C, Marzouk K, Sharma O: Sarcoidosis of the spinal cord: literature review and report of eight cases. J Nat Med Assoc 2006;98:965-976. 